\title{
Achieving Subnanometer Precision in a MEMS-Based Storage Device During Self-Servo Write Process
}

\author{
Abu Sebastian, Member, IEEE, Angeliki Pantazi, Member, IEEE, S. O. Reza Moheimani, Senior Member, IEEE, \\ Haris Pozidis, Member, IEEE, and Evangelos Eleftheriou, Fellow, IEEE
}

\begin{abstract}
In probe-based data storage devices, microelectromechanical system-based microscanners are typically used to position the storage medium relative to the read/write probes. Global position sensors are employed to provide position information across the full scan range of these microscanners. However, to achieve repeatable positioning, it is also necessary to have medium-derived position information. Dedicated storage fields known as servo fields are employed to obtain this medium-derived position information. The servo-patterns on these servo fields have to be written using the global position sensors prior to the regular operation of the storage device by employing a scheme known as "self-servo write" process. During this process, subnanometer positioning resolutions, well below that provided by the global position sensors, are desirable. Such precise positioning at acceptable bandwidth requires the directed design of the closed-loop noise sensitivity transfer function so as to minimize the impact of sensing noise. This paper describes control architectures in which the impact of measurement noise on positioning is minimal while providing satisfactory tracking performance. It is estimated that the positioning error due to sensing noise is a remarkably low $0.25 \mathrm{~nm}$. Experimental results are also presented that show error-free operation of the device at high densities.
\end{abstract}

Index Terms $-\mathrm{H}_{\infty}$ controllers, nanopositioning, precision positioning, probe-based data storage, resonant controllers, servo write process.

\section{INTRODUCTION}

$\mathbf{S}$ CANNING-PROBE data storage technology is considered as an ultrahigh density and low-power alternative to conventional data storage. A probe-storage device uses nanometersharp tips, as they are typically used in scanning probe microscopes, to record and readback data. Such a technology can be regarded as a natural candidate for overcoming the physical limits on achievable areal density in conventional storage technologies.

One concept of a probe-based storage device is presented in [1]-[3]. A schematic of the probe-based storage device is shown in Fig. 1. This device is based on a thermomechanical

Manuscript received January 7, 2008. First published June 20, 2008; current version published September 26, 2008. The review of this paper was arranged by Associate Editor L. Dong.

A. Sebastian, A. Pantazi, H. Pozidis, and E. Eleftheriou are with the International Business Machines (IBM) Zurich Research Laboratory, Rüschlikon 8803, Switzerland (e-mail: ase@zurich.ibm.com; agp@zurich.ibm.com; hap@zurich.ibm.com; ele@zurich.ibm.com).

S. O. R. Moheimani is with the School of Electrical Engineering and Computer Science, University of Newcastle, Callaghan, NSW 2308, Australia (e-mail: reza.moheimani@newcastle.edu.au).

Color versions of one or more of the figures in this paper are available online at http://ieeexplore.ieee.org.

Digital Object Identifier 10.1109/TNANO.2008.926441

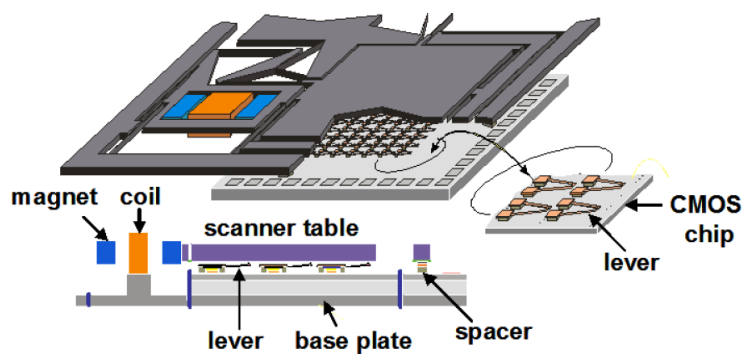

Fig. 1. Schematic of the probe-based storage device. Reprinted from [1].

principle for storing and retrieving information written on thin polymer films. Owing to the relatively low data rates per probe, a microelectromechanical (MEMS)-based array of probes are operated in parallel with each probe performing read/write/erase operations on an individual storage field. Digital information is stored by making indentations on the thin polymer film by means of the tips of microcantilevers, which are a few nanometers in diameter. The shape of a typical indentation resembles an almost conical structure with a diameter of approximately $15-30 \mathrm{~nm}$. This indentation shape results in an error-rate performance that rapidly deteriorates as the probe-tip distance from the center of the indentation increases [4]. Hence, nanopositioning is a key enabling technology.

Displacement of the storage medium relative to the array of microcantilevers is achieved by using a silicon-based microscanner with $x / y$-motion capabilities greater than $100 \mu \mathrm{m}$ [5]. The $x / y$-positional information of the microscanner is provided by global position sensors integrated on the device and known as thermal position sensors [6]. Even though these thermal sensors have satisfactory noise performance at high frequencies, they tend to suffer from significant low-frequency drift owing to ambient temperature variations. There could even be sensitivity changes over the lifetime of the device, rendering a feedback-control scheme relying on the thermal sensors alone unsuitable for long-term operation of the device. Hence, for absolute positioning in a $100 \mu \mathrm{m} \times 100 \mu \mathrm{m}$ storage field with nanometer-scale resolution, one has to rely on some form of medium-derived position information besides the thermal position sensors. Because multiple probes are available, a small number of probes and their storage fields could be dedicated for the generation of the medium-derived positional information. Such track-seeking and track-following controllers that utilize thermal sensors and the medium-derived positional information have been designed and successfully implemented [7]. 
Prior to using a MEMS-based scanning-probe storage device, the servo patterns generating the medium-derived positional information have to be written in those storage fields reserved for this specific purpose. This operation is referred to as servo writing. Note that in contrast to hard disk drives, the servo information is written without assistance from an external positioning device [8]. Hence, it is called "self-servo write" process. Subnanometer-scale positioning accuracies are desired while performing self-servo write operation as the positioning errors incurred while writing the servo fields would appear as sensing noise while performing regular read/write operation of the device. Open-loop operation is not possible during self-servo write operation primarily because of the lightly damped poles of the microscanner. Conventional feedback controllers on the other hand would make the system sensitive to measurement noise, particularly the low-frequency components, and sensor drift.

To overcome this problem, we introduce the concept of shaping the noise sensitivity transfer function as applied to the "selfservo write" application. When controlling a dynamical system, it is imperative that there be frequency regions in which "control is essential", e.g., the resonance frequency region in the case of the microscanner. It is also evident that in these frequency regions, the closed-loop system would be sensitive to measurement noise. However, it is highly desirable and, as shown in this paper, possible to carefully design the noise sensitivity transfer function such that the closed-loop system is insensitive to measurement noise outside these frequency regions. In nanopositioning applications that require positioning errors to be well below the resolution provided by the position sensors, a directed shaping of the noise sensitivity transfer function is essential. Control architectures for controlling the microscanner along both the $x$ - and $y$-scan directions based on $H_{\infty}$ controllers and resonant controllers are presented that provide us with the desired noise sensitivity transfer functions.

The remainder of the paper is organized as follows. In Section II, a brief description of the essential components of the positioning system is provided. The control architectures and the analysis of closed-loop performance are presented in Section III. Experimental results obtained from a probe-based data storage prototype, demonstrating the efficacy of the control architecture, are presented in Section IV.

\section{Positioning System}

\section{A. Components}

The microscanner that is used to position the storage medium relative to the read/write probes has $x / y$-motion capabilities that are on the order of the pitch between microcantilevers in the array (see Fig. 2). The scan table, which carries the polymer storage medium, can be displaced in two orthogonal directions $(x$ and $y)$ in the plane of the silicon wafer. Two pairs of thermal position sensors are used to provide $x / y$-position information of the microscanner. Thermal position sensors are microheaters that form part of the chip carrying the cantilever array (see Fig. 2). They are placed in such a way that they partially overlap the scan table. As the scanner moves, there is a change in the overlap between the sensors and the scan table, which results

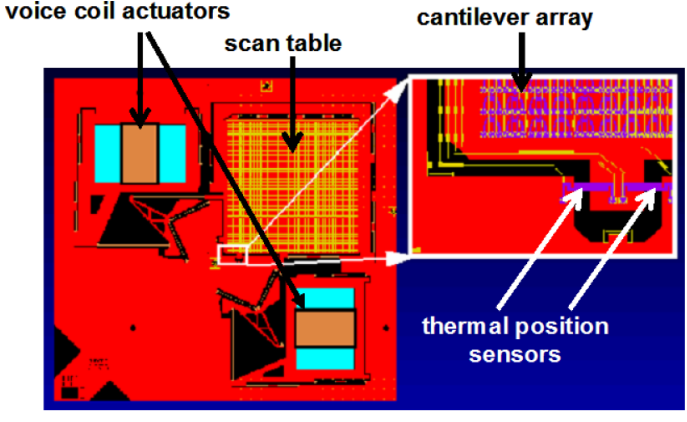

Fig. 2. Schematic of the microscanner and thermal position sensors.

in a change in their temperature. This change in temperature is detected electrically owing to the resulting change in the electrical resistance. These differential sensors are remarkably linear over the travel range of the microscanner and, as will be seen later, have resolutions of approximately $1 \mathrm{~nm} 1 \sigma$ over the sensing bandwidth of approximately $5 \mathrm{kHz}$.

Next, we describe the medium-derived positional information, the generation of which requires the self-servo write process. Certain storage fields and their respective levers are assigned to the generation of this position error signal (PES) [see Fig. 3(a)]. The method to generate this medium-derived PES is based on the concept of sequences of indentations (bursts) that are mutually displaced vertically, arranged in such a way as to produce two signals in quadrature, which can be combined to provide a position error signal [1], [7]. Medium-derived PES can be generated using the servo burst configuration illustrated in Fig. 3(b), where the circles represent written indentations recorded on four different storage fields. The servo bursts are recorded prior to the regular operation of the device in the servo fields. Servo bursts labeled A and B are used to create the inphase signal $(I)$, and $\mathrm{C}$ and $\mathrm{D}$ the quadrature signal $(Q)$. $I$-signal is obtained by subtracting the readback signal from the B field from that from the A field, whereas the $Q$-signal is obtained by subtracting the readback signal from the $\mathrm{D}$ field from that from the $\mathrm{C}$ field. The cross-track distance between indentation centers of the same burst is equal to the track pitch (TP), whereas the distance between indentation centers in bursts $\mathrm{A}$ and $\mathrm{B}$ (or $\mathrm{C}$ and $\mathrm{D})$ is $\mathrm{TP} / 2$. The distance between the $\mathrm{A}$ and $\mathrm{C}$ centers is $\mathrm{TP} / 4$. During regular operation of the device, the data are recorded in the storage fields aligned to the sequence of indentations in burst $\mathrm{C}$. In other words, the track centerlines of the data fields coincide with the corresponding centerlines of burst C. Note that the $Q$-signal exhibits zero crossings at points where the $I$-signal has local extrema, as shown in Fig. 3(c). A signal that has zero crossings at all track center locations and a linear range between $-\mathrm{TP} / 2$ and TP/2 can be generated by combining the two signals $(I$ and $Q)$. The medium-derived PES provides $y$-positional information around each track centerline, and therefore, has a maximum range of $\mathrm{TP}$.

\section{B. Identification and Modeling}

A comprehensive model of the microscanner was developed for control design and simulation purposes. The device 


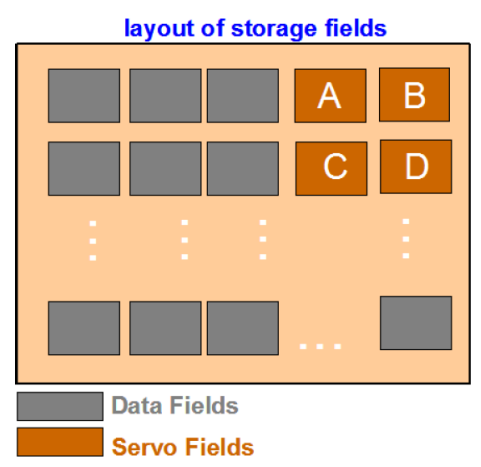

(a)

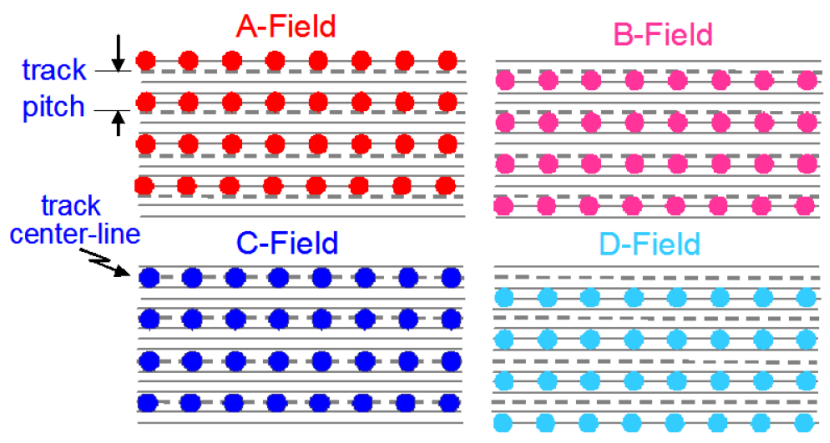

(b)

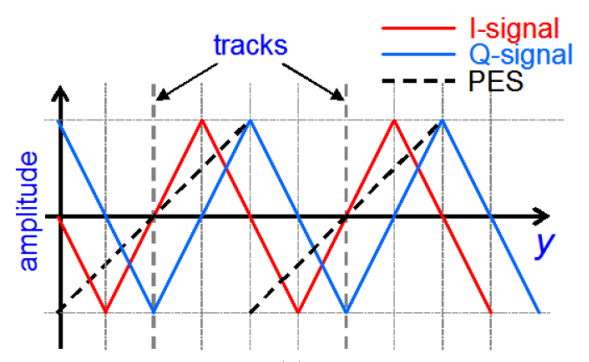

(c)

Fig. 3. Schematics depicting the servo fields and the generation of mediumderived PES.

is viewed as a two-input two-output system. The motion of the microscanner in each direction is modeled by the linear dynamic components $P_{x x}$ and $P_{y y}$. Specifically, the transfer function $P_{x x}$ models the mapping from the coil current ( $u_{x}$ in milliampere) to the output displacement $x$ (in millimeter), and the transfer function $P_{y y}$ models the mapping from the coil current $u_{y}$ to the output displacement $y$. The cross-coupling between the axes is modeled as the sum of the linear dynamic components $P_{x y}$ and $P_{y x}$ with the memoryless nonlinear functions of the scanner positions $x$ and $y, f(x, y)$, and $g(x, y)$, respectively. However, in the frequency region of interest, the linear dynamic components of the cross-coupling $P_{x y}$ and $P_{y x}$ are found to be relatively small compared with the nonlinear components.

To identify the transfer functions $P_{x x}$ and $P_{y y}$, the frequency responses of the scanner in the $x$-and $y$-directions are obtained using the thermal position sensors in the range from $1 \mathrm{~Hz}$ to $10 \mathrm{kHz}$. The frequency responses of the $x$ - and $y$-scanner axes are shown in Fig. 4. As can be seen, the dynamics is dominated by the first mode. However, the second-order model does not fully describe the behavior of the microscanner in the
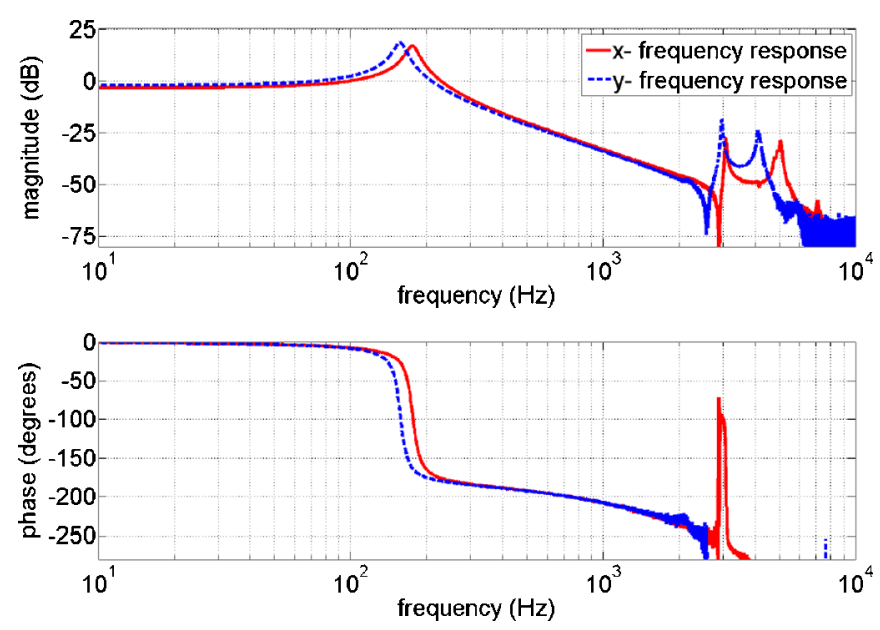

Fig. 4. Experimentally obtained frequency response of the scanner motion in the $x$ - and $y$-scan directions as measured by the sensor.

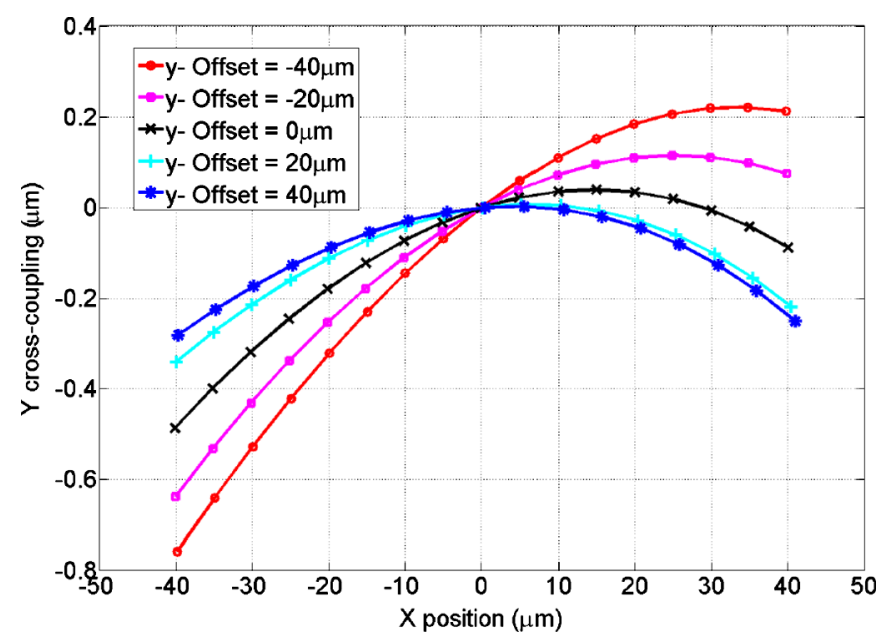

Fig. 5. Cross-coupling between the $x$ - and $y$-axes.

higher frequency regime, where it exhibits higher order resonance modes. It is also found that these higher order resonances change with the position on the $x y$ plane, which further complicates an exact modelling of the microscanner. Fig. 5 shows the cross-coupling on the $y$-axis due to $x$-motion for various values of displacement in the $y$-scan direction. As shown in this figure, the cross-coupling is nonlinear and position dependent.

The thermal position sensor bandwidth is determined by the thermoelectric response of the heaters. Typically, a first-order response with a time constant of around 60-100 $\mu$ s accurately captures the sensor dynamics. Schemes for the experimental identification of the thermoelectrical response of these microheaters are described in [9]. Positioning accuracy is related to the intrinsic noise characteristics of the sensors. The total measurement noise comprises the thermal position sensor noise and the quantization noise from the 16-bit analog-to-digital converters (ADCs) used. The standard deviation of the combined noise has been measured to be $0.97 \mathrm{~nm}$ over $10 \mathrm{kHz}$ bandwidth for the $x$-sensor and $1.00 \mathrm{~nm}$ for the $y$-sensor. Although the accuracy of the sensor is reasonably good, there is a significant 


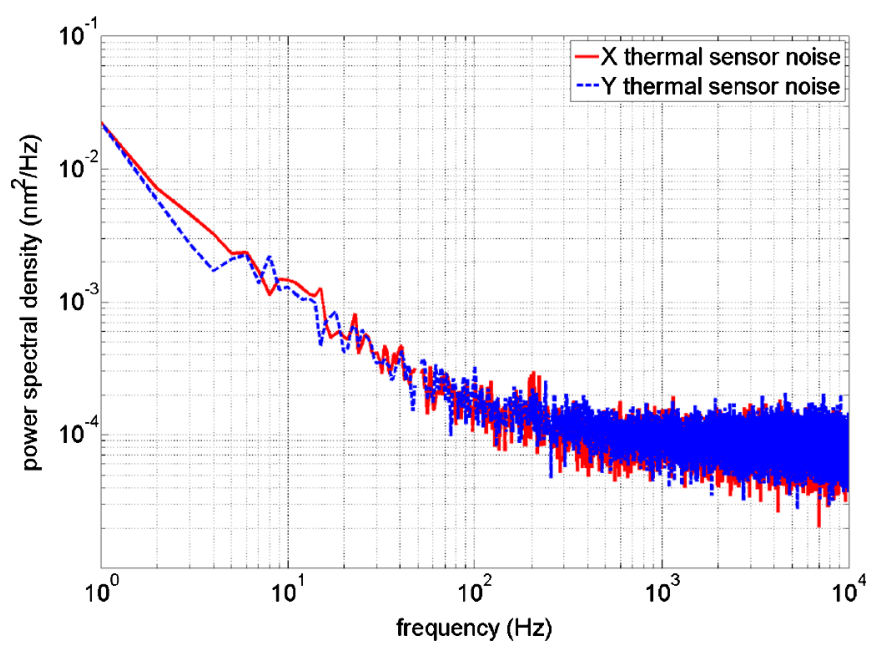

Fig. 6. Measured power spectral density of thermal-sensor noise.

low-frequency component as evident from the power spectral density of the sensor noise shown in Fig. 6. Moreover, these sensors are susceptible to drift and minor sensitivity variations.

\section{Controller Design for Self-Servo Writing}

During the self-servo write operation, only the thermal position sensors are available to provide position information. We have argued that significantly high accuracies are desired while writing servo fields because positioning errors incurred while recording the servo patterns translate to measurement noise in the PES during regular device operation. The tolerable positioning error is well below the resolution of the thermal position sensors. Hence, as discussed earlier, the control design should be such that the closed-loop system is sensitive to measurement noise only in the frequency regions where control is essential. Moreover, as evident from Fig. 6, the thermal-sensor measurements are particularly noisy at low frequencies. It is essential that the closed-loop system does not suffer significantly from sensor noise, drift, and other low-frequency noises. The immunity to drift is particularly important. It is also essential that the closed-loop system has an acceptable reference tracking bandwidth. These requirements translate to desired shapes for the closed-loop noise sensitivity and reference-tracking transfer functions. In subsequent sections, this directed shaping of these key transfer functions is illustrated.

\section{A. $x$-Control Architecture}

The control architecture for the $x$-scan direction is presented in this section. The control architecture for the $y$-scan direction is similar to that of the $x$-scan direction. However, owing to the cross-coupling between the two scan axes, additional steps are required in the design of the control architecture for the $y$-scan direction. This will be presented later.

The block diagram describing the feedback loop for the $x$-axis is illustrated in Fig. 7. There, $r_{x}$ is the reference signal during the self-servo write operation (typically a triangular wave); $w_{x}$ represents the input disturbance signals to the plant, namely

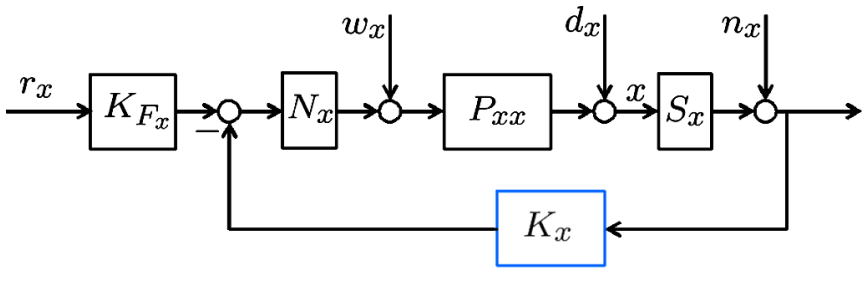

Fig. 7. Block diagram describing the feedback loop for the $x$-scan direction.

shocks and vibrations as well as actuator noise; $d_{x}$ represents output disturbance signals; and $n_{x}$ represents the sensor noise. The filter $N_{x}(s)$ represents a set of notch filters centered at the out-of-bandwidth resonances of the microscanner. These notch filters are incorporated into the feedforward loop to minimize the effect of the two out-of-bandwidth resonances on the feedbackcontrolled system. The feedforward block $K_{F_{x}}$ is merely a gain equivalent to the inverse of the dc gain of $P_{x x}(s)$. Furthermore, $S_{x}(s)$ represents the low-pass dynamics of the thermal position sensors. The primary component of the control architecture is, however, the feedback controller $K_{x}$. It is designed to meet the performance measures described earlier, which are captured in terms of requirements on the appropriate closed-loop transfer functions.

The closed-loop transfer functions are powerful tools for characterizing the performance of controllers [10]. From the block diagram shown in Fig. 7, the closed-loop transfer functions $T_{x r}$, $T_{x n}, T_{x d}$, and $T_{x w}$, are given by

$$
\begin{aligned}
& T_{x r}(s)=\frac{K_{F_{x}}(s) N_{x}(s) P_{x x}(s)}{1+K_{x}(s) N_{x}(s) P_{x x}(s) S_{x}(s)} \\
& T_{x n}(s)=\frac{-K_{x}(s) N_{x}(s) P_{x x}(s)}{1+K_{x}(s) N_{x}(s) P_{x x}(s) S_{x}(s)} \\
& T_{x d}(s)=\frac{1}{1+K_{x}(s) N_{x}(s) P_{x x}(s) S_{x}(s)} \\
& T_{x w}(s)=\frac{P_{x x}(s)}{1+K_{x}(s) N_{x}(s) P_{x x}(s) S_{x}(s)} .
\end{aligned}
$$

They denote the transfer functions relating the position with the reference, sensing noise, output disturbance, and input disturbance, respectively.

While designing $K_{x}$, the main objective is to obtain a desirable noise sensitivity transfer function $T_{x n}$. The requirement on acceptable reference tracking performance is captured using $T_{x r}$. The requirements on $T_{x d}$ and $T_{x w}$ are minimal because the self-servo write process is typically performed under controlled conditions, such as a vibration-free environment. Two approaches are presented in the subsequent sections. One approach is to pose the problem in the framework of $H_{\infty}$ control. The other approach is to employ resonant controllers, which is possible because the microscanner resembles a flexible structure with a collocated sensor and actuator pair.

\section{B. $H_{\infty}$ Controller Design}

In this section, we illustrate the use of the $H_{\infty}$ control framework for the design of $K_{x}$. The block diagram in Fig. 8 shows 


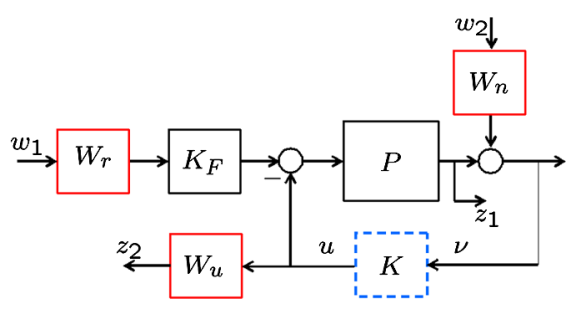

Fig. 8. Block diagram depicting the formulation for $H_{\infty}$ control design.

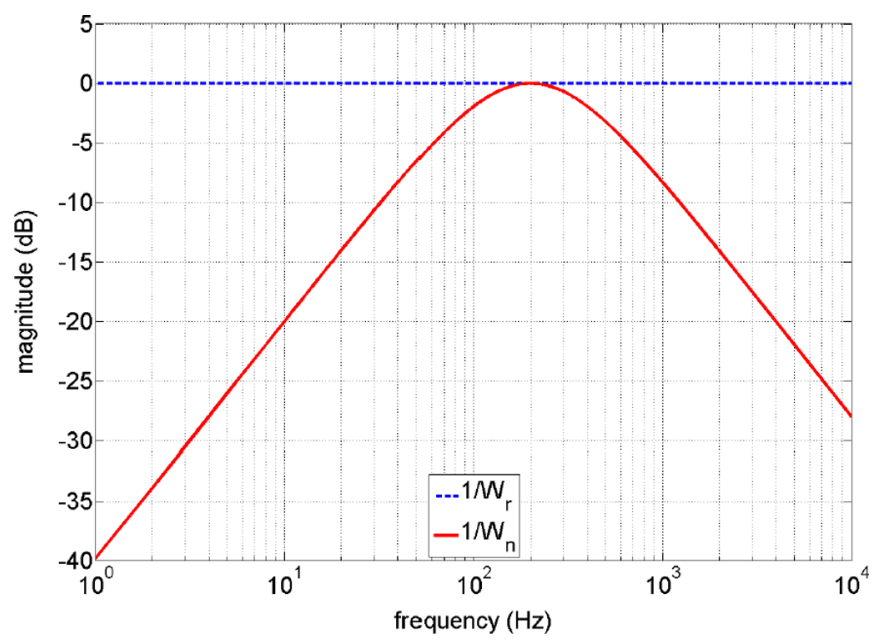

Fig. 9. Magnitude responses of the noise and reference weighting functions.

the formulation of the control design problem. $P$ denotes the model of the microscanner used for the control design. Typically, a lower order approximation of the identified models $P_{x x}$ or $P_{y y}$ is used for control design.

Linear systems known as weighting functions $W_{r}$ and $W_{n}$ are used to capture the requirements on reference tracking and sensitivity to measurement noise, respectively. To limit the use of control effort, a weighting function $W_{u}$ is also introduced. The magnitude responses of $W_{r}$ and $W_{n}$ are shown in Fig. 9. The selection of $W_{r}$ to be equal to 1 ensures damping of the scanner dynamics and good transient behavior. The response of $1 / W_{n}$ captures the desired magnitude response of the closedloop noise sensitivity transfer function. The bandpass nature of $1 / W_{n}$ centered around the resonance frequency of the scanner dynamics captures the requirement for the closed-loop system to be sensitive to measurement noise only where control is essential. The low-frequency roll-off captures the requirement to be less sensitive to low frequency noise. $W_{n}$ was chosen to be

$$
W_{n}=\frac{0.5 s^{2}+1257 s+7.9 \times 10^{5}}{0.001 s^{2}+1257 s+1579}
$$

and $W_{u}$ was chosen to be $1 / 10$.

The signals $w_{1}$ and $w_{2}$ are known as "exogenous inputs" and $z_{1}$ and $z_{2}$ are known as "exogenous outputs." The "sensed output" and "control signal" are denoted by $v$ and $u$, respectively. The $H_{\infty}$ control design problem is to find a stabilizing controller $K$ such that $\left\|T_{z w}\right\|_{\infty}<\gamma_{\infty}$, where $\gamma_{\infty}$ is a constant approximately equal to 1 [11]. Here, $T_{z w}$ is the closed-loop

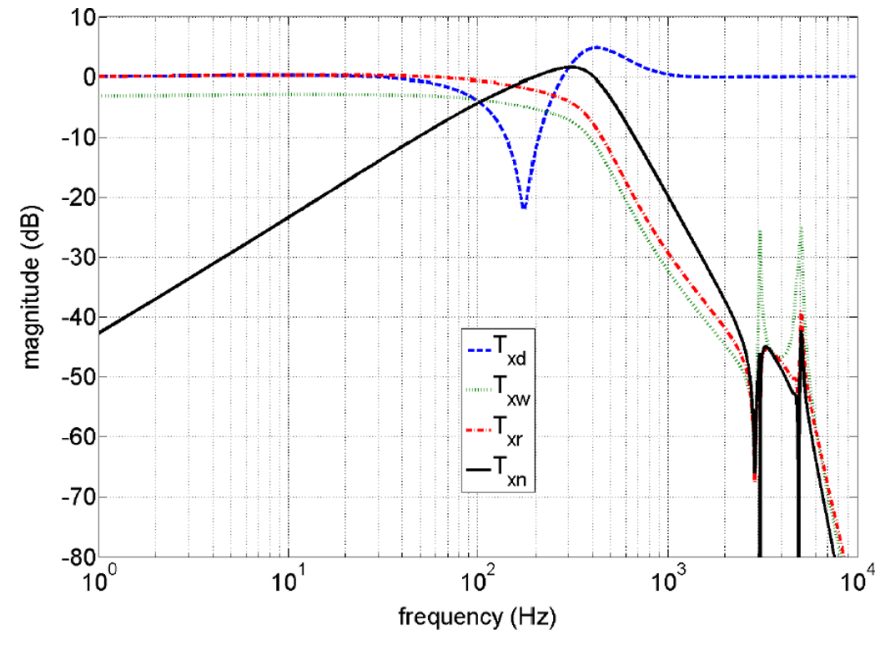

Fig. 10. Closed-loop transfer functions corresponding to the $x$-axis and the $H_{\infty}$ controller.

transfer function matrix relating $z$ with $w$, where

$$
z=\left[\begin{array}{l}
z_{1} \\
z_{2}
\end{array}\right] \quad \text { and } \quad w=\left[\begin{array}{l}
w_{1} \\
w_{2}
\end{array}\right] \text {. }
$$

From the discussion in Section II-B, it can be deducted that the dynamics of the microscanner, along $x$ - and $y$-directions, are well captured by that of a spring-mass-damper second-order model. The high-frequency resonances of the microscanner, which tend to vary as a function of the microscanner position, can be neglected at the design stage. Using the second-order approximation of the scanner dynamics and the weighting functions described earlier, fourth-order controllers are obtained. For the $x$ - and $y$-scan directions, the controllers are obtained to be

$$
\begin{aligned}
& K_{x}(s)=\frac{67682.88\left(s+1.26 \times 10^{6}\right)(s-18.81)(s+1.26)}{\left(s+2.73 \times 10^{6}\right)(s+16.84)\left(s^{2}+6733 s+2.11 \times 10^{7}\right)} \\
& K_{y}(s)=\frac{431.6\left(s+1.26 \times 10^{6}\right)(s+1275)(s+1.257)}{\left(s+1.72 \times 10^{4}\right)(s+1275)\left(s^{2}+6388 s+2.09 \times 10^{7}\right)} .
\end{aligned}
$$

Note that in the $H_{\infty}$ design approach, there is no limitation on the nature of $P$ chosen for control design. It is possible to use higher order approximations of $P_{x x}$ and $P_{y y}$. It is also possible to incorporate the sensor dynamics. The weighting functions can also be chosen to incorporate a priori spectral characteristics of measurement noise and of the reference signals that need to be tracked. However, using higher order system models and weighting functions will result in higher order controllers.

Fig. 10 shows the magnitude response of $T_{x r}(s), T_{x n}(s)$, $T_{x d}(s)$, and $T_{x w}(s)$ corresponding to the $K_{x}$ designed using the $H_{\infty}$ approach. All closed-loop transfer functions display a sharp damping at and close to the resonance frequency of the microscanner. This is clearly due to the control action. It can be seen that the reference tracking transfer function $T_{x r}$ and the noise sensitivity transfer function $T_{x n}$ are shaped by the infinity norm constraints imposed using the weighting functions $W_{r}$ and $W_{n}$, respectively. The transfer function $T_{x n}$ is shaped such that the closed-loop system is sensitive to measurement noise only in the region where control is essential. 
TABLE I

PARAMETERS OF $P_{x x}$ AND $P_{y y}$

\begin{tabular}{|c|c|c|}
\hline & $x x$ & $y y$ \\
\hline$\alpha$ & 0.7 & 0.8 \\
\hline$\zeta$ & 0.0484 & 0.0388 \\
\hline$\omega$ & 1108.5 & 983.4 \\
\hline
\end{tabular}

\section{Resonant Controller Design}

Another controller that provides the desired noise sensitivity transfer function is the resonant controller. Unlike the $H_{\infty}$ control design approach, such controllers are mainly designed for collocated dynamical systems. However, they have a very simple structure, do not require a higher bandwidth than that of the system being controlled, and can guarantee closed-loop stability in presence of out-of-bandwidth dynamics. The second-order approximations of the microscanner dynamics along $x$ - and $y$-scan directions, which were used in the design of the $H_{\infty}$ controllers, can be represented as

$$
\frac{\alpha_{x x} \omega_{x x}^{2}}{s^{2}+2 \zeta_{x x} \omega_{x x} s+\omega_{x x}^{2}} \text { and } \frac{\alpha_{y y} \omega_{y y}^{2}}{s^{2}+2 \zeta_{y y} \omega_{y y} s+\omega_{y y}^{2}}
$$

respectively, where all parameters are shown in Table I. It is clear that these transfer functions, which correspond to the first resonant mode of the microscanner along $x$ - and $y$-scan directions represent collocated systems. A number of controller design methodologies have been developed for highly resonant systems with collocated sensors and actuators. Positive position feedback control (PPF) [12] and the recently-developed positive position and velocity feedback control (PVPF) [13] are two examples of the controllers designed for collocated systems. An alternative approach to efficiently damp a collocated system is to use a resonant controller. Resonant controllers have been shown to be very efficient in terms of adding damping to flexible structures with collocated sensors and actuators [14], [15]. What makes them particularly attractive for our application is their performance in terms of measurement noise.

For a single-mode, single-input single-output (SISO) system, a resonant controller is defined as

$$
K(s)=\frac{\gamma \tilde{\omega}^{2} s^{2}}{s^{2}+2 \delta \tilde{\omega} s+\tilde{\omega}^{2}}
$$

and can be designed in a number of ways. It is possible to minimize a cost function, such as $H_{2}$, or $H_{\infty}$ norm of the system as a function of controller parameters. This generally results in a nonlinear optimization problem, whose solution can be determined numerically [15]. However, because of their simple structure, such controllers can usually be designed by inspection. In particular for collocated SISO systems with low modal density, this approach generally works quite well. Here, the resonant controllers $K_{x}(s)$ and $K_{y}(s)$ were designed by inspection to control the microscanner's motion along the $x$ - and $y$-axes, respectively. Controller parameters are tabulated in Table II.

Fig. 11 shows the magnitude response of transfer functions $T_{x r}(s), T_{x n}(s), T_{x d}(s)$, and $T_{x w}(s)$ corresponding to $K_{x}$ designed using the resonant controller approach. As in the case of the $H_{\infty}$ design, $T_{x n}(s)$, has a bandpass profile, centered at the first resonance frequency of the microscanner. The sensitivity
TABLE II

PARAMETERS OF $K_{x}$ AND $K_{y}$

\begin{tabular}{|c|c|c|}
\hline & $x x$ & $y y$ \\
\hline$\gamma \tilde{\omega}^{2}$ & 3.0 & 3.0 \\
\hline$\delta$ & 1.1 & 1.1 \\
\hline$\tilde{\omega}$ & 1108.5 & 983.4 \\
\hline
\end{tabular}

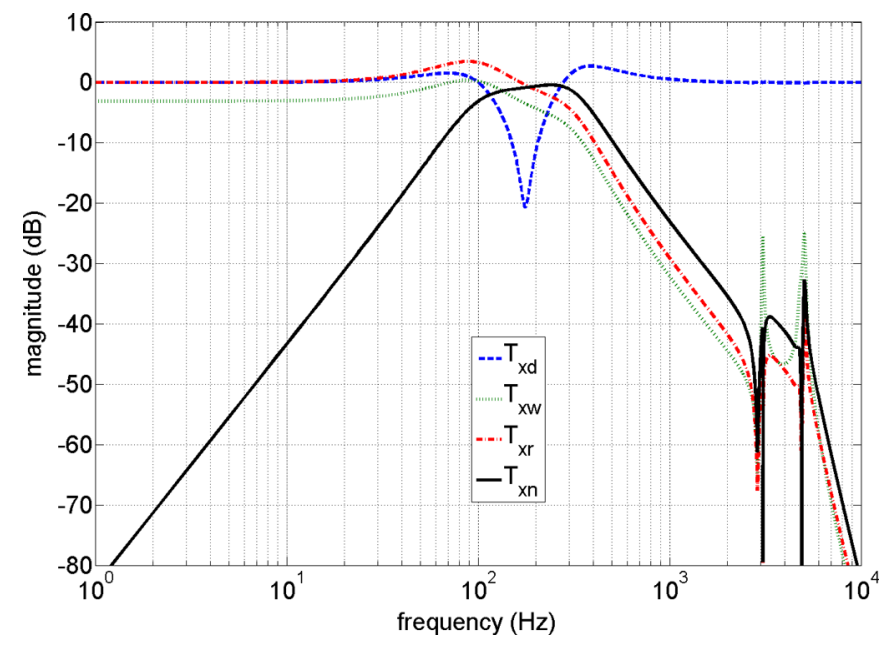

Fig. 11. Closed-loop transfer functions corresponding to the $x$-axis and the resonant controller.

to measurement noise is limited to the frequency region where control over the dynamics is essential, i.e., the resonance frequency and its vicinity. Moreover, it has a very desirable $40 \mathrm{~dB}$ per decade roll-off at the low- and high-frequency regions. This stems from the fact that $K_{x}(s)$ has a double zero at the origin, which substantially reduces the effect of low-frequency sensor noise on the closed-loop system.

In both approaches, apart from adding damping to the microscanner, the controller reduces the effect of sensor noise on the position of the microscanner to an absolute minimum. The price that is paid is in terms of $T_{x d}$ and $T_{x w}$. The closed-loop system is very sensitive to input and output disturbances. In particular, low- and high-frequency output disturbances directly affect the position of microscanner. However, the effect of such disturbances at and close to the resonance frequency of the microscanner is significantly reduced thanks to the introduced damping. Furthermore, note that input and output disturbances do not pose a significant problem as the self-servo write operation is performed in a controlled vibration-free environment.

\section{D. y-Control Architecture}

The $y$-axis feedback loop, as depicted in Fig. 12, is very similar to the $x$-axis loop. However, its function is rather different. The reference signal for the $y$-scan direction $r_{y}$ is fixed for each period of the triangular signal applied to $r_{x}$ to facilitate the task of servo writing over a straight line on the storage medium. However, the cross-coupling from the $x$-axis on the $y$-axis during a scan is quite substantial, as discussed earlier in Section II-B. Ideally, the feedback controller for $y$-axis positioning should be able to damp the microscanner's $y$-axis dynamics, shape the noise transfer function along the lines explained earlier, and 


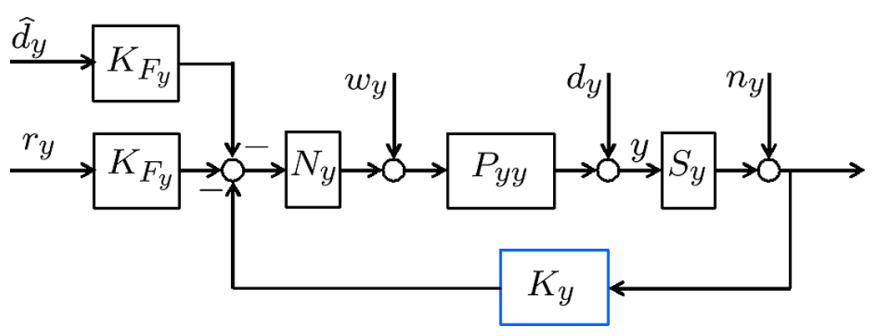

Fig. 12. Block diagram describing the feedback loop for the $y$-scan direction.

reduce the effect of output disturbances on the $y$-position at in-bandwidth frequencies. However, the last two objectives are contradictory. It is straightforward to show that the two transfer functions of interest satisfy the condition

$$
T_{y d}(s)=1+S_{y}(s) T_{y n}(s) .
$$

Therefore, they cannot be made arbitrarily small over a given frequency region. Note that $S_{y}(s)$, the sensor transfer function, has a unity gain and negligible phase at in-bandwidth frequencies.

The controller $K_{y}$ is designed, as described earlier, using any of the two approaches. It damps the first resonant mode of the microscanner in the $y$-axis and shapes the noise transfer function as required. However, because of condition (6), the $y$-position of the microscanner remains sensitive to cross-coupling from scanning along the $x$-axis. The fact that the controller hardly corrects for output disturbance signals at low frequencies is now used to reduce the effect of these cross-couplings. Writing of servo fields is performed in two stages with the $y$-axis set point held constant. First, a line is scanned and the $y$-axis position as measured by the $y$-axis thermal sensor is recorded. The recorded information provides an excellent estimate of the cross-coupling signal over the scanning range; and consequently, the signal $\hat{d}$ in Fig. 12 can be determined as follows:

$$
\hat{d}_{y}=\frac{d_{y}}{1+K_{y}(s) N_{y}(s) P_{y y}(s) S_{y}(s)} .
$$

Clearly, this cross-coupling estimate is not free of sensor noise. However, it is safe to assume that the low-frequency noise component of the thermal position sensor signal in the period of just one scan is insignificant. The high-frequency noise could be eliminated by filtering. The same scan is then repeated by applying $\hat{d}_{y}$ as a feedforward signal, as shown in Fig. 12. For low-frequency scans, this strategy results in minimal residual error. This procedure is then repeated for all tracks over which the servo fields are to be written. Although this doubles the time needed for completion of the self-servo write process, it results in writing of servo fields in a satisfactory manner, as illustrated experimentally in the next section.

\section{EXPERIMENTAL RESULTS AND DISCUSSION}

First, experimental results are presented using the control architectures for the self-servo write process. Then, we present results from the regular operation where medium-derived PES from the servo patterns written, using the self-servo write process is employed. The controllers were implemented on a Texas
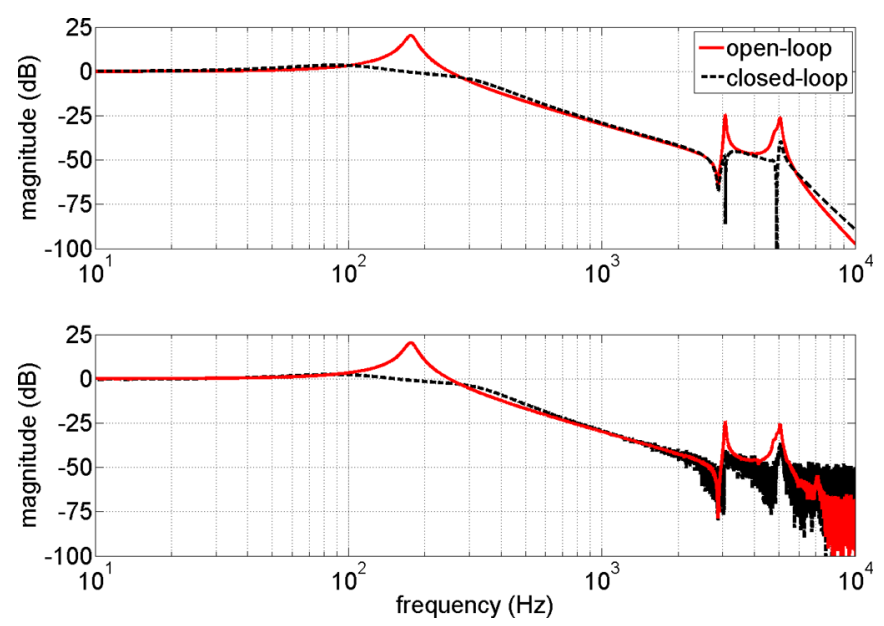

Fig. 13. Theoretical (top) and experimental (bottom) open- $\left(P_{x x}\right)$ and closedloop $\left(T_{x r}\right)$ transfer functions.

Instruments TMS320C6713 digital signal processing (DSP). The sampling frequency was chosen to be the relatively high, $50 \mathrm{kHz}$, to minimize the impact of quantization noise. The 16-bit ADCs were used to sample the thermal position sensor signal and 16-bit digital-to-analog converters (DACs) were used to output the control signal.

\section{A. Self-Servo Write Process}

The control architectures described in Section III were simulated using the models developed for the storage device. The controllers $K_{x}$ and $K_{y}$ designed using both the design approaches showed satisfactory performance. However, owing to the simplicity, superior low-frequency roll-off behavior of the noise sensitivity transfer function and dc measurement noise insensitivity, $K_{x}$ and $K_{y}$ designed using the resonant controller approach were chosen for implementation.

The frequency responses of the open-loop model $P_{x x}$ and of the closed-loop model $T_{x r}$ (obtained using (1)) are plotted in Fig. 13. It can be seen that there is a good agreement between the analytical and experimental results. It can also be seen that the controller adds $18 \mathrm{~dB}$ damping to the microscanner's first resonant mode. This amounts to shifting the open-loop poles of the microscanner located at $-53.7 \pm j 1107.2$ to $-319.3 \pm$ $j 556.3$ and $-953.7 \pm j 1661.6$ in the closed loop. The increased damping improves the tracking performance significantly. In the $x$-scan direction, this enables higher scan velocities, and in the $y$-scan direction, it mitigates the impact of transients while stepping from track to track.

The frequency response of the operator relating the $x$-position with the $x$-output disturbance is obtained experimentally. This is achieved by artificially creating a disturbance signal (chirp or white noise) and feeding it into the closedloop system. This frequency response is compared with that of the analytically evaluated $T_{x d}$ transfer function using (3) (see Fig. 14). The high sensitivity of the closed-loop system to disturbance signals is clearly visible. In Fig. 14, a similar comparison is also made between the frequency response of the operator relating the $x$-position with the $x$-measurement noise and that 

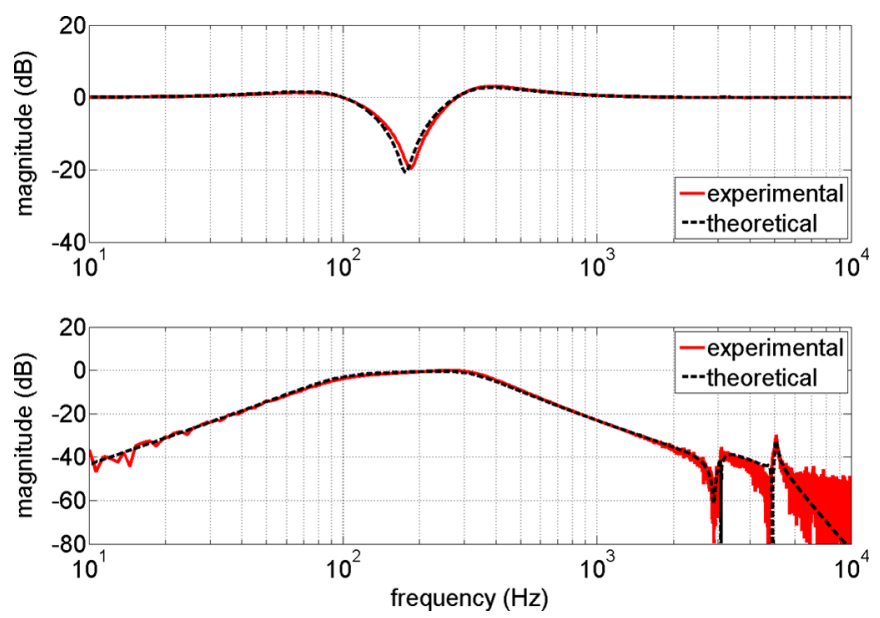

Fig. 14. Comparison of experimentally obtained and calculated $T_{x d}$ (top) and $T_{x n}$ (bottom) transfer functions.

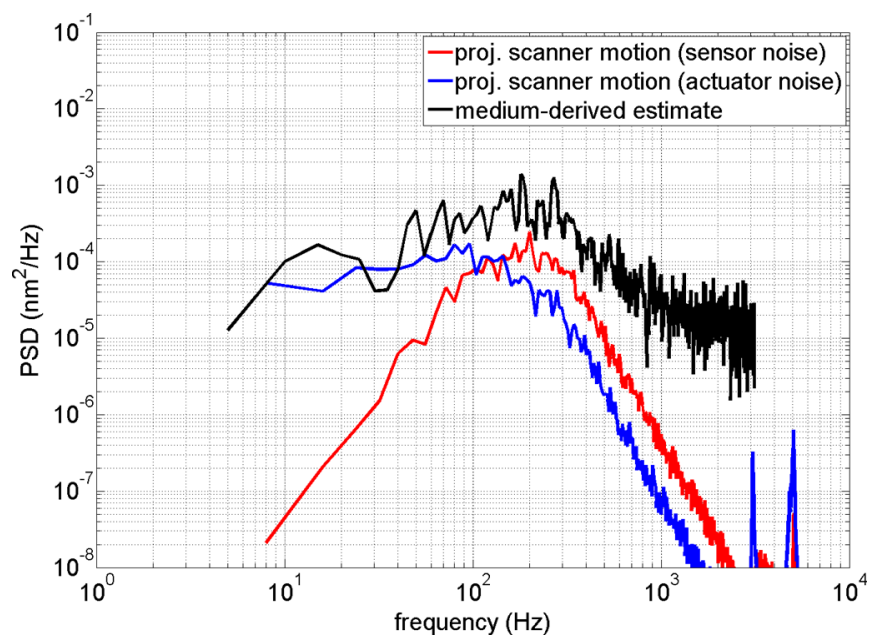

Fig. 15. Demonstration of the impact of measurement noise and actuator quantization noise on the positioning resolution.

of the $T_{x n}$ transfer function evaluated using (2). The main attribute of the control architecture, namely, selective sensitivity to measurement noise, is clearly visible.

From the closed-loop transfer functions, the positioning accuracy of the closed-loop system can be characterized. Two significant non-deterministic contributors to positioning error while performing servo writing are sensing noise and actuator quantization noise. The $1 \sigma$ sensor noise was measured to be $0.97 \mathrm{~nm}$ over $10 \mathrm{kHz}$. From $T_{x n}$, one could estimate the impact of sensor noise on positioning, which is evaluated to be $0.21 \mathrm{~nm} 1 \sigma$ (see Fig. 15). The actuator noise, which includes the quantization noise from the 16-bit DAC, is estimated to be $1.1 \mu \mathrm{A} 1 \sigma$ over $10 \mathrm{kHz}$. The impact of actuator noise is estimated using $T_{x w}$. It is found that the positioning error introduced is less than $0.16 \mathrm{~nm} 1 \sigma$. Hence, the net positioning error due to the stochastic components is estimated to be an astonishingly low $0.25 \mathrm{~nm}$.

In a regular nanopositioning device, it is difficult to verify the positioning accuracy derived using the closed-loop transfer functions. However, the nanoscale manipulation capability of

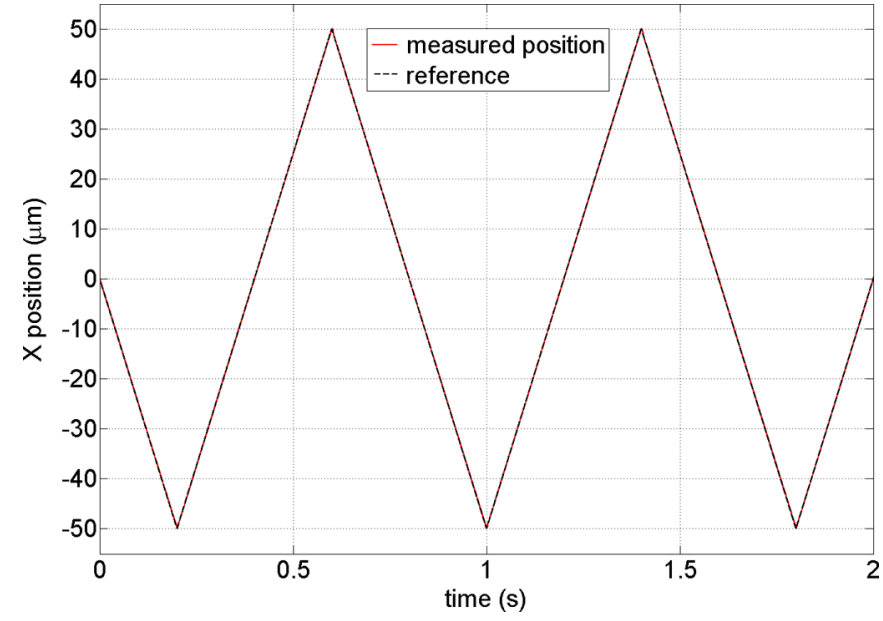

Fig. 16. Measurements obtained from $x$-sensor during the self-servo write process.

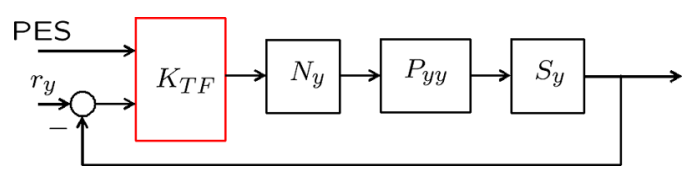

Fig. 17. Two-sensor-based track follow controller employed during regular device operation.

the microcantilevers provides a unique opportunity to verify the positioning accuracy. Indentations could be formed at regular intervals by a micro-cantilever while scanning using the proposed control architecture. The deviations of the indentation centers from their desired locations could be measured. This provides an alternative way to characterize the positioning accuracy [16]. Even though this estimate is rather noisy, the spectral characteristic of the media-derived estimate closely resembles the estimate of the positioning errors obtained from the closed-loop transfer functions (see Fig. 15). The $x$-sensor measurement, while performing a repeated forward/backward 100- $\mu$ m-long scan operation, is shown in Fig. 16. The scan linear velocity is $0.25 \mathrm{~nm} / \mu \mathrm{s}$. Because of the increased damping, there are no oscillations near the turn-around points.

\section{B. Read-Write Demonstration}

The performance of the controller is further illustrated in a complete read/write demonstration based on the mediumderived PES obtained from the servo patterns written using the self-servo write process. Four microcantilevers were chosen to write the servo fields using the proposed control architecture. After this write process, the medium-derived PES obtained from those servo fields was used to control the scanner along the $y$-scan direction using a two-sensor-based track-following controller, as shown in Fig. 17, denoted by $K_{T F}$. Details of this scheme are reported in [7] and [17]. The operation was performed while random data were being read from four data levers. The data bits were recorded with an indentation pitch of $56 \mathrm{~nm}$ and symbol pitch of $28 \mathrm{~nm}$. The readback signals from the four servo fields are shown in Fig. 18(a). It can be seen 


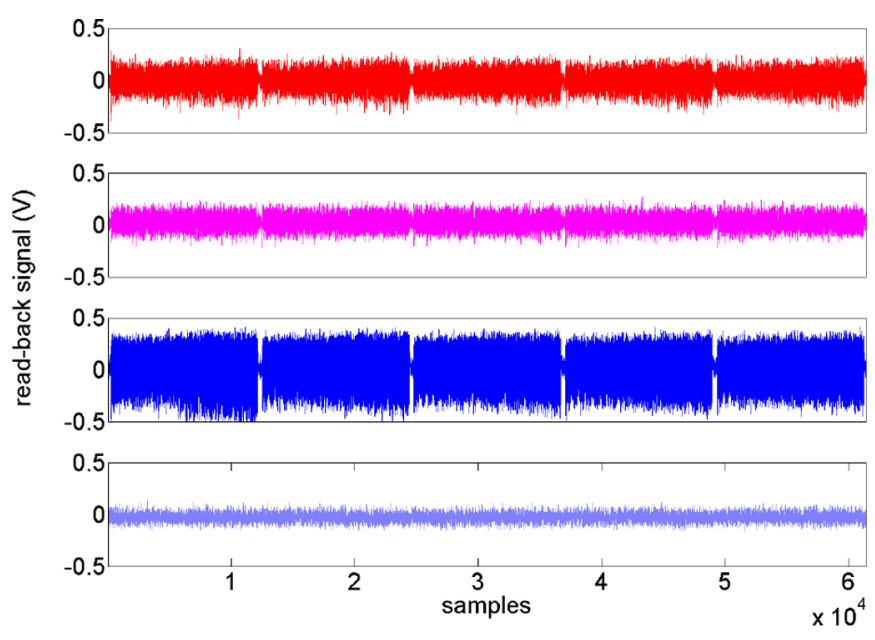

(a)

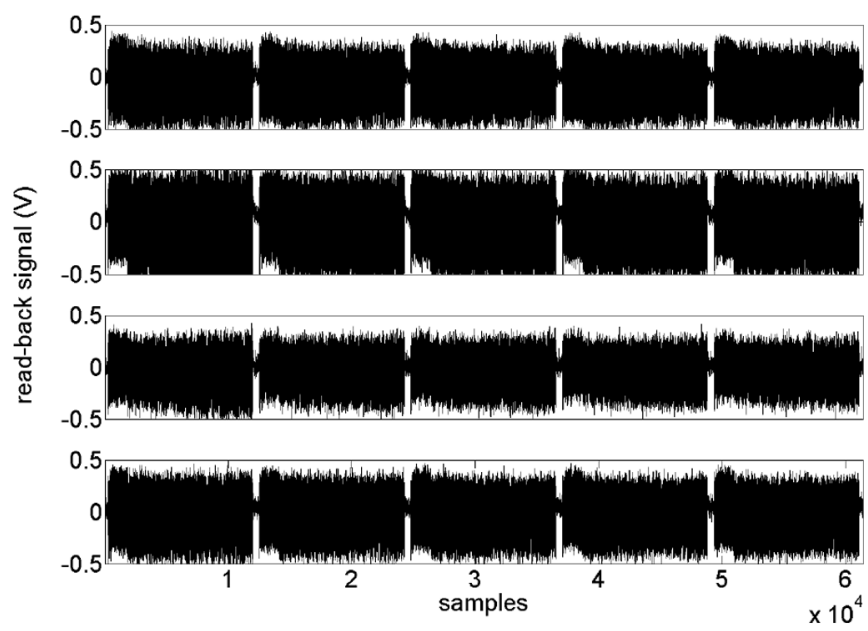

(b)

Fig. 18. (a) Read-back signals from the four servo levers, A, B, C and D. (b) Read-back signals from the four data levers.

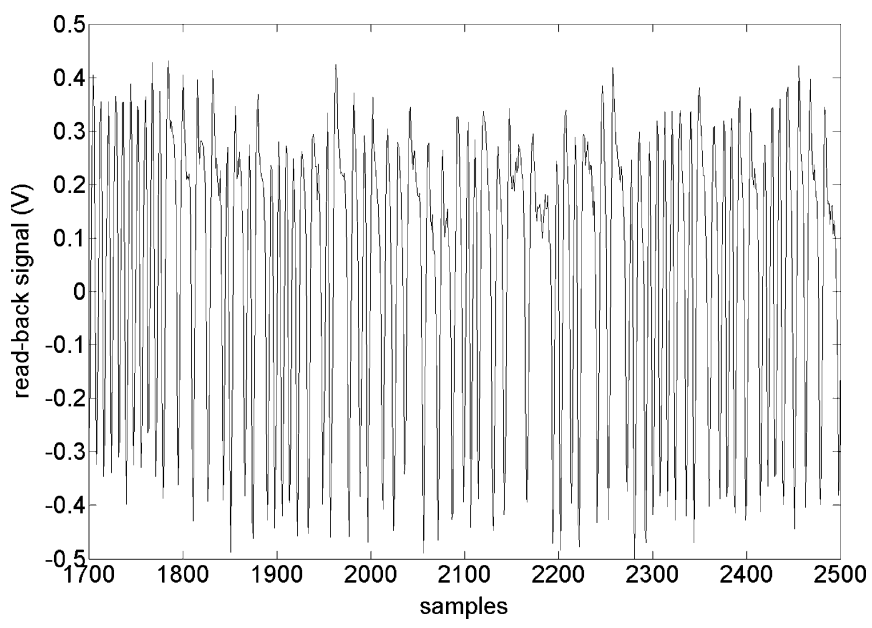

Fig. 19. Close-up of the signal from the data fields. that the signal from the $\mathrm{C}$ field is the strongest whereas there is hardly any signal from the $\mathrm{D}$ field. The signal strength in the A and $\mathrm{B}$ fields is equal. This implies that the levers are on track in the data fields because the data fields are written aligned to the $\mathrm{C}$ field. The readback signals from the four data fields are shown in Fig. 18(b). Owing to the remarkable accuracy with which the servo patterns were recorded and the associated high quality of the medium-derived PES signal, no errors were detected in this $\mathrm{read} /$ write demonstration comprising over 60000 bits.

\section{CONCLUSION}

MEMS-based scanning-probe data storage devices are emerging as ultrahigh density, low-power alternatives to conventional storage devices. The probe-based data storage concept involving thermomechanical storage on thin polymer media is arguably the most advanced scanning-probe-based storage scheme. A small-scale storage device prototype based on this concept has been developed, which consists of an array of microcantilevers each recording information on storage fields $100 \mu \mathrm{m} \times$ $100 \mu \mathrm{m}$ in size. The ultrahigh areal densities that these devices can deliver impose stringent requirements on the positioning accuracies needed. An MEMS microscanner with 2-D motion capability is employed to position the storage medium relative to the cantilever array. A pair of thermal position sensors per axis provide position information across the travel range of the microscanner. However, to achieve repeatable positioning over the large storage area, dedicated servo fields are employed to obtain medium-derived position information. These servo fields have to be written prior to the operation of the device by means of a process known as self-servo write. subnanometer positioning resolutions are needed while writing these servo fields. Such precise positioning at acceptable bandwidth using the thermal position sensors requires directed design of the closed-loop noise sensitivity transfer function so as to minimize the impact of sensing noise. Control architectures are introduced for both $x$ - and $y$-scan directions that have the desired closed-loop noise sensitivity transfer function. Experimental results are presented that show the remarkable positioning accuracies that can be achieved. The positioning error due to actuator noise and sensing noise is estimated to be around $0.25 \mathrm{~nm}$. servo fields are written using the proposed control architecture. Experimental write/read results based on the medium-derived position information further demonstrate the efficacy of the control scheme.

\section{ACKNOWLEDGMENT}

The authors thank their colleagues of the Probe Storage Group at the IBM Zurich Research Laboratory. They also thank M. Despont, U. Drechsler, M. Lantz, and H. Rothuizen for the design and fabrication of MEMS parts, D. Jubin for the characterization and assembly of the MEMS components, and P. Bächtold for the design of the electronics used in this paper. They would also like to thank C. Bolliger for assistance with the preparation of the manuscript. 


\section{REFERENCES}

[1] E. Eleftheriou, T. Antonakopoulos, G. K. Binnig, G. Cherubini, M. Despont, A. Dholakia, U. Dürig, M. A. Lantz, H. Pozidis, H. E. Rothuizen, and P. Vettiger, "Millipede-a MEMS based scanning-probe data storage system," IEEE Trans. Magn., vol. 39, no. 2, pp. 938-945, Mar. 2003.

[2] P. Vettiger, G. Cross, M. Despont, U. Drechsler, U. Dürig, B. Gotsmann, W. Häberle, M. Lantz, H. Rothuizen, R. Stutz, and G. Binnig, "The 'millipede'-nanotechnology entering data storage," IEEE Trans. Nanotechnol., vol. 1, no. 1, pp. 39-55, Mar. 2002.

[3] A. Pantazi, A. Sebastian, T. Antonakopoulos, P. Bächtold, T. Bonaccio, J. Bonan, G. Cherubini, M. Despont, R. A. Dipietro, U. Drechsler, U. Dürig, W. Häberle, C. Hagleitner, J. L. Hedrick, D. Jubin, A. Knoll, M. A. Lantz, J. Pentarakis, H. Pozidis, R. C. Pratt, H. Rothuizen, R. Stutz, M. Varsamou, D. Wiesmann, and E. Eleftheriou, "Probe-based ultra-high density storage technology," IBM J. Res. Dev., vol. 52, no. 4/5, 2008.

[4] H. Pozidis, W. Häberle, D. W. Wiesmann, U. Drechsler, M. Despont, T. Albrecht, and E. Eleftheriou, "Demonstration of thermomechanical recording at 641 Gbit / in ${ }^{2}$," IEEE Trans. Magn., vol. 40, no. 4, pp. 25312536, Jul. 2004.

[5] M. A. Lantz, H. Rothuizen, U. Drechsler, W. Haeberle, and M. Despont, "A vibration resistant nanopositioner for mobile parallel-probe storage applications," J. Microelectromech. Syst., vol. 16, no. 1, pp. 130-139, Feb. 2007.

[6] M. A. Lantz, G. K. Binnig, M. Despont, and U. Drechsler, "A micromechanical thermal displacement sensor with nanometer resolution," Nanotechnology, vol. 16, pp. 1089-1094, May 2005.

[7] A. Pantazi, A. Sebastian, G. Cherubini, M. Lantz, H. Pozidis, H. Rothuizen, and E. Eleftheriou, "Control of MEMS-based scanningprobe data-storage devices," IEEE Trans. Control Syst. Technol., vol. 15, no. 5, pp. 824-841, Sep. 2007.

[8] A. Al Mamun, G. Guo, and B. Chao, Hard Disk Drive : Mechatronics and Control, 1st ed. Boca Raton, FL: CRC, 2006.

[9] A. Sebastian and D. Wiesmann, "Modeling and experimental identification of silicon microheater dynamics: A systems approach," J. Microelectromech. Syst., vol. 17, no. 4, pp. 911-920, Aug. 2008.

[10] A. Sebastian and S. Salapaka, "Design methodologies for robust nanopositioning," IEEE Trans. Control Syst. Technol., vol. 13, no. 6, pp. 868876, Nov. 2005.

[11] S. Skogestad and I. Postlethwaite, Multivariable Feedback Control: Analysis and Design, 2nd ed. New York: Wiley/Interscience, 2005.

[12] J. L. Fanson and T. K. Caughey, "Positive position feedback-control for large space structures," AIAA J., vol. 28, no. 4, pp. 717-724, Apr. 1990.

[13] B. Bhikkaji, M. Ratnam, and S. O. R. Moheimani, "PVPF control of piezoelectric tube scanners," Sens. Actuators A, Phys., vol. 135, no. 2, pp. 700-712, Apr. 2007.

[14] S. O. R. Moheimani and B. J. G. Vautier, "Resonant control of structural vibration using charge-driven piezoelectric actuators," IEEE Trans. Control Syst. Technol., vol. 13, no. 6, pp. 1021-1035, Nov. 2005.

[15] D. Halim and S. O. R. Moheimani, "Spatial resonant control of flexible structures-application to a piezoelectric laminate beam," IEEE Trans. Control Syst. Technol., vol. 9, no. 1, pp. 37-53, Jan. 2001.

[16] A. Sebastian, A. Pantazi, and H. Pozidis, "Jitter investigation and performance evaluation of a small-scale probe storage device prototype," in Proc. IEEE Global Commun. Conf., Washington, DC, Nov. 26-30, 2007, pp. 288-293.

[17] A. Pantazi, A. Sebastian, H. Pozidis, and E. Eleftheriou, "Two-sensorbased $\mathrm{H}_{\infty}$ control for nanopositioning in probe storage," in Proc. IEEE Conf. Decis. Control, Seville, Spain, Dec. 12-15, 2005, pp. 1174-1179.

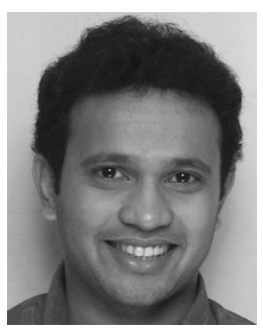

Abu Sebastian (M'03) received the B.E. (Hons.) degree in electrical and electronics engineering from Birla Institute of Technology and Science, Pilani, India, in 1998, and the M.S. and Ph.D. degrees in electrical engineering from Iowa State University, Ames, in 1999 and 2004, respectively.

$\mathrm{He}$ is currently a Research Staff Member at IBM's Zurich Research Laboratory, Rüschlikon, Switzerland. Recently, he was involved in the development of the first scanning-probe-based data storage device prototype.

His primary research interests include dynamics and control at the nanometer scale. Research interests include microcantilever-based devices and enabling technologies like nanometer-scale sensing and nanopositioning.

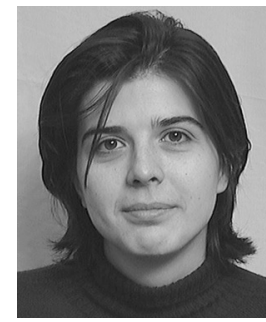

Angeliki Pantazi (M'06) received the Diploma and $\mathrm{Ph} . \mathrm{D}$. degrees in electrical engineering and computer technology from the University of Patras, Patras, Greece, in 1996 and 2005, respectively.

She is currently a Research Staff Member at the IBM Zurich Research Laboratory, Rüschlikon, Switzerland. Her current research interests include probe-based technologies with emphasis on nanopositioning, system-level design for probe-based datastorage devices, and magnetic tape drive systems.

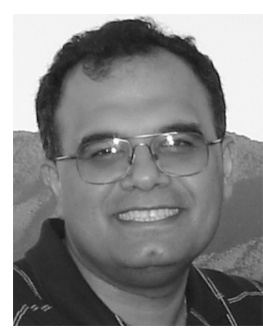

S. O. Reza Moheimani (SM'00) received the Ph.D. degree in electrical engineering from the University of New South Wales at the Australian Defence Force Academy, Canberra, Australia, in 1996.

$\mathrm{He}$ has held several visiting appointments at IBM Zurich Research Laboratory, Switzerland. He is currently a Professor at the School of Electrical Engineering and Computer Science, University of Newcastle, Callaghan, Australia, where he serves as the Assistant Dean Research for the Faculty of Engineering and Built Environment. He is also an Associate Director of the ARC Centre for Complex Dynamic Systems and Control, an Australian Government Centre of Excellence. He is the author or coauthor of two published books, several edited volumes, and over 150 refereed articles in archival journals and conference proceedings. His current research interests include applications of control and estimation in nanoscale positioning systems for scanning probe microscopy, and control of electrostatic microactuators in microelectromechanical systems and data storage systems.

Prof. Moheimani is a Fellow of the Institute of Physics, U.K. He has served on the Editorial Board of a number of journals including the IEEE TRANSACTIONS ON CONTROL SYSTEMS TECHNOLOGY, and has chaired several international conferences and workshops. He was a recipient of the 2007 IEEE TRANSACTIONS ON CONTROL SYstems TECHNOLOGY Outstanding Paper Award.

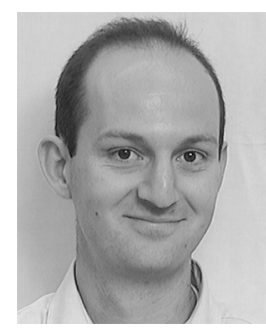

Haralampos Pozidis (M'98) received the Diploma in computer engineering and informatics from the University of Patras, Greece, in 1994, and the M.Sc. and $\mathrm{Ph} . \mathrm{D}$. degrees in electrical engineering from Drexel University, Philadelphia, PA, in 1997 and 1998, respectively.

From 1998 till 2001, he was with Philips Research Eindhoven, The Netherlands, where he was engaged in research on read channel design for optical storage devices, in particular digital video disk and Blu-ray disk formats, with focus on channel modeling, equalization, and bit detection. Since 2001, he has been with the IBM Zurich Research Laboratory, Rüschlikon, Switzerland, where he has been engaged in research on basic recording technology, signal processing, and overall system design for microelectromechanical-system-based scanning probe data storage devices.

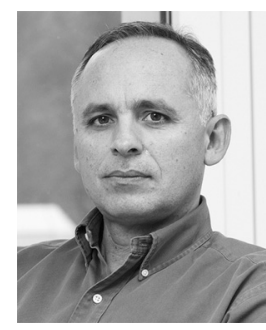

Evangelos Eleftheriou (F'02) received the B.S. degree in electrical engineering from the University of Patras, Patras, Greece, in 1979, and the M. Eng. and $\mathrm{Ph} . \mathrm{D}$. degrees in electrical engineering from Carleton University, Ottawa, ON, Canada, in 1981 and 1985, respectively.

He joined the IBM Zurich Research Laboratory, Rüschlikon, Switzerland, in 1986, where he was engaged in research on various projects related to wired and wireless communications, magnetic recording, and probe storage. He currently manages the laboratory's Advanced Storage Technologies Group.

Dr. Eleftheriou became an IBM Fellow and was elected to the IBM Academy of Technology in 2005. He was a corecipient of the Eduard Rhein Technology Award in 2005. He was a corecipient of the 2003 IEEE Communications Society Leonard G. Abraham Prize Paper Award. 